\title{
MORTALIDADE MATERNA POR CAUSAS OBSTÉTRICAS NO ESTADO DA PARAÍBA
}

\author{
Helloysa Karynna Sttefanny de Oliveira e Silva' \\ Ana Carolina Almeida Pereira" \\ Smalyanna Sgren da Costa Andrade ${ }^{\text {III }}$
}

\section{RESUMO}

A mortalidade materna é um agravo que, apesar de ser em sua maioria por causas evitáveis, ainda se apresenta como um grave problema de saúde pública. Esta pesquisa objetivou analisar a mortalidade materna por causas obstétricas. Trata-se de um estudo ecológico, retrospectivo e de abordagem quantitativa, realizado no período de junho de 2019. O estudo identificou que as mortes maternas se apresentam de forma mais elevada por causas diretas, dentro do grupo de mulheres menos alfabetizadas, pardas e solteiras. Existe a necessidade de uma melhor assistência profissional, de forma qualificada e holística, tendo em vista a vulnerabilidade social das mulheres frente ao risco de mortalidade materna. Assim, concluiu-se que o pré-natal qualificado é fator determinante para a redução dessas mortes maternas por causas obstétricas no estado da Paraíba.

PALAVRAS-CHAVE: Saúde da Mulher. Obstetrícia. Saúde Pública. Enfermagem.

Enfermeira. Pós-graduanda em Obstetrícia e Neonatologia. João Pessoa, Paraíba. Brasil. I ORCID: 0000-0001-6378-0137

Enfermeira. Pós-graduanda em Enfermagem Obstétrica. João Pessoa, Paraíba, Brasil. Autora correspondente. E-mail: carolinaalmeidajp@gmail.com ORCID ID: 0000-0002-2949-1988

Enfermeira. Doutora em Enfermagem pela Universidade Federal da Paraíba, Docente do curso de Graduação em Enfermagem e do Mestrado Profissional em Saúde da Família da FACENE/FAMENE, 58067-695, João Pessoa, Paraíba. Brasil. ORCID ID: 0000-0002-8732-5080 


\section{INTRODUÇÃO}

A Organização Mundial da Saúde (OMS) entende por morte materna o eventual óbito de uma mulher durante a gestação, ou que esteja dentro do período puerperal que se estende até 42 (quarenta e dois) dias depois do término do ciclo gestacional. Portanto, tal complicação deve estar relacionada, ou agravada pela gestação. Aquelas complicações e óbitos ocasionados por acidentes e/ou incidentes não se enquadram no termo morte materna. ${ }^{1}$

Anualmente, em todo o mundo, estipula-se que cerca de 500.000 mulheres morrem de complicações gestacionais e do parto. Cerca de sete mil mulheres que sobrevivem a essas complicações são diagnosticadas com sérios problemas de saúde e quase 50 mil sofrem eventos adversos à saúde em consequência do parto. Vale salientar que a maioria dessas doenças e complicações ocorrem nos países em desenvolvimento ${ }^{2}$. Nota-se, que mesmo havendo um número elevado de morte materna, por causas obstétricas, ainda não foi possível identificar a real dimensão dos sub-registros e subnotificações desses óbitos e as causas reais dessas mortes com destaque para os países subdesenvolvidos. $^{2}$

Observou-se em outro estudo que, no Brasil, o índice de mortalidade materna decorrentes de causas obstétricas apresenta oscilações dentre as regiões, porém, na região Centro-Oeste houve maior prevalência. Além disso, o não preenchimento satisfatório das mortes maternas por causas obstétricas é um problema a ser combatido. Um dos estudos destaca a importância de atitudes de alteridade por parte dos gestores, na intenção de diminuir essas subnotificações, registrando devidamente as causas obstétricas de mortalidade materna
VOLUME 17 - NÚMERO 2 - AGO/2019 ISSN ELETRÔNICO 2317-7160 para que o resultado seja mais preciso e demonstre maior veracidade. 3

As causas da mortalidade materna podem ser causas obstétricas diretas, ou seja, aquelas resultantes de alterações que comprometem o ciclo gravídico-puerperal, que podem estar relacionadas diretamente com medidas assistenciais incorretas e desqualificadas, causando prejuízos significativos na gravidez, parto e puerpério. Já as causas obstétricas indiretas são resultantes de complicações no histórico prévio da genitora, podendo também se agravar durante o ciclo gravídico, em consequência das alterações fisiológicas atribuídas pela gestação e não advindas de causas diretas ${ }^{4}$. Considera-se que a maioria das causas diretas são preveníveis e as causas indiretas estão relacionadas às mulheres acometidas por doenças e devem, necessariamente, ser consideradas, de início, como gestantes de risco e acompanhadas em maior complexidade ${ }^{2}$.

Desse modo, considerando o impacto da mortalidade entre gestantes no Brasil e no mundo, bem como compreendendo a necessidade de averiguação do comportamento epidemiológico desta problemática, a fim de perceber modificações relevantes ao longo dos anos, entre as gestantes do Estado da Paraíba, e sugerir uma resposta coletiva dos gestores frente ao contexto evidenciado, esta pesquisa foi norteada pelo seguinte questionamento: Qual a tendência anual, étnica, instrucional e de situação conjugal frente à mortalidade entre gestantes de um estado nordestino? Para tanto, objetivou-se analisar os casos de mortalidade materna por causas obstétricas diretas e indiretas entre gestantes paraibanas. 


\section{MATERIAL E MÉTODOS}

Trata-se de estudo ecológico, retrospectivo e de abordagem quantitativa. A coleta dos dados ocorreu no mês de junho de 2019, com uso de dados secundários disponíveis a acesso público. Para tanto, o estudo foi desenvolvido por meio dos dados provenientes do Departamento de Informática do Sistema Único de Saúde (DATASUS).

O local-alvo da pesquisa foi o Estado da Paraíba. Foram uzadas as informações do ano de 2013 a 2017 para mortalidade materna. A sequência operacional da consulta, após acessar o site oficial do DATASUS, para mortalidade materna foi: acesso à informação, informações de saúde (TABNET), estatísticas vitais, mortalidade - 1996 a 2017 pela CID-10; e por último, os óbitos de mulheres em idade fértil e óbitos maternos. Como a pesquisa utilizou dados secundários do Ministério da Saúde do Brasil, logo não necessitou de tramitação em Comitê de Ética em Pesquisa, em conformidade com a Resolução n ${ }^{\circ}$ 466/2012 do Conselho Nacional de Saúde. 5

As variáveis foram as causas diretas e indiretas de mortalidade materna por ano. $\mathrm{O}$ dados foram analisados por meio de estatística descritiva através de frequência absoluta (número) e relativa (percentual) e expostos em forma de tabela.

\section{RESULTADOS E DICUSSÃO}

A tendência temporal da mortalidade materna na Paraíba, por causas obstétricas diretas e indiretas, pode ser visualizada na Tabela
1. Os resultados demonstraram que as causas diretas de mortalidade materna são mais pre valentes que as causas indiretas em todos os

TABELA 1. Distribuição da mortalidade materna de mulheres paraibanas por causa obstétrica direta ou indireta entre 2013-2017. João Pessoa, Paraíba, Brasil. 2019. ( $N=192)$

\begin{tabular}{|c|c|c|c|c|c|c|}
\hline \multirow{2}{*}{ Variáveis } & \multirow{2}{*}{$\mathrm{CO}$} & \multicolumn{5}{|c|}{ Ano } \\
\hline & & 2013 & 2014 & 2015 & 2016 & 2017 \\
\hline \multirow{2}{*}{ Gravidez, parto ou aborto } & D & $14(7,0 \%)$ & $8(4,0 \%)$ & $12(6,0 \%)$ & $6(3,0 \%)$ & $5(14,3)$ \\
\hline & I & - & $3(1,5 \%)$ & $1(0,5 \%)$ & $2(1,0 \%)$ & $5(14,3)$ \\
\hline \multirow{2}{*}{ Puerpério até 42 dias } & D & $8(4,0 \%)$ & $22(11,1 \%)$ & $8(4,0 \%)$ & $19(9,6 \%)$ & $14(40,0 \%)$ \\
\hline & I & $1(0,5 \%)$ & $4(2,0 \%)$ & $3(1,5 \%)$ & $5(2,5 \%)$ & $6(17,1 \%)$ \\
\hline \multirow{2}{*}{ Puerpério 43 dias à 1 ano } & D & $1(0,5 \%)$ & - & $1(0,5 \%)$ & - & $1(2,9 \%)$ \\
\hline & 1 & - & - & - & - & - \\
\hline \multirow{2}{*}{ Não na gravidez ou puerpério } & D & $1(0,5 \%)$ & - & $2(1,0 \%)$ & - & - \\
\hline & I & $2(1,0 \%)$ & - & - & - & - \\
\hline \multirow{2}{*}{ Não informado/ignorado } & D & $24(12,5 \%)$ & $1(0,5 \%)$ & $4(2,0 \%)$ & $3(1,5 \%)$ & $3(8,6 \%)$ \\
\hline & I & $3(1,5 \%)$ & - & - & - & $1(2,9 \%)$ \\
\hline Subtotal & & $48(24,1 \%)$ & $31(15,6 \%)$ & $27(13,6 \%)$ & $28(14,6 \%)$ & $12(34,3 \%)$ \\
\hline Total & & 54 & 31 & 35 & 37 & 35 \\
\hline
\end{tabular}


anos investigados. Além disso, as maiores diferenças de ocorrências para causas diretas e indiretas estiveram relacionadas ao puerpério até 42 dias nos anos ímpares. Cabe mencionar que o contraste entre estas duas causas também ocorreu para dados relacionados a gravidez, parto ou abortamento, cuja oscilação anual apontou os anos pares.

No Brasil, as principais causas de óbitos maternos registrados em 2010 foram por causas obstétricas diretas. Entre as causas com maior impacto na ocorrência destes óbitos estão a hipertensão (19,7\%), a hemorragia $(10,9 \%)$ a infecção puerperal $(6,5 \%)$ e o abortamento (4,6\%). Em relação aos óbitos por causas indiretas, as doenças do aparelho circulatório complicadas pela gestação, parto ou puerpério foram os eventos mais frequentes, repercutindo-se em 7,6\% do total de óbitos. 3

Segundo dados da Organização Pan-Americana de Saúde (OPAS), cerca de 830 muIheres morrem todos os dias por complicações relacionadas a gravidez, ou ao parto em todo o mundo. Estima-se que em 2015 cerca de 303 mil mulheres morreram durante e após a gravidez e o parto. Mesmo após uma década, a mortalidade materna ainda apresenta numerações elevadas e tornou-se um grande alerta para a saúde, começando a se pensar em uma forma melhor e mais humanizada para o nascimento do bebê. 6

Além disso, também se pode acrescentar uma qualidade frágil na assistência prestada a mulher, do pré-natal até o puerpério, configurando-se como fatores condicionantes e determinantes à saúde da mulher na gestação e pós-gestação. ${ }^{4}$ A Política de Atenção Integral a Saúde da Mulher (PAISM) foi criada na tentativa de humanizar o pré-natal de qualidade. Suas práticas de saúde holísticas são elementos importantes a redução dos índices de mortalidade materna no Brasil. A formação profissional aliada aos programas de redução de vulnerabilidades sociais de instâncias governamentais são fatores contribuintes a melhoria do cuidado obstétrico e neonatal.7

Em 2013, o programa de Residência em Enfermagem Obstétrica ganhou força no Brasil, sendo inserido como estratégia política da Rede Cegonha. A intenção do governo federal era a melhoria da assistência pré-natal, parto e pós-parto das mulheres que utilizam o Sistema Único de Saúde. ${ }^{8}$ Neste ano de 2013 , a prevalência de subnotificação reduziu na Paraíba, ao passo que a quantidade de mortes por causas obstétricas cresceu em comparação ao ano anterior. Talvez esse resultado possa ser reflexo da inserção de profissionais especialistas na área, nos diversos setores e serviços de saúde obstétricos, gerando identificação e parâmetros reais sobre as causas de morte materna no estado.

Na Tabela 2 é possível observar a prevalência de mortalidade materna conforme a quantidade total de anos estudados.

TABELA 2. Distribuição da mortalidade materna de mulheres paraibanas por escolaridade entre 2013-2017. João Pessoa, Paraíba, Brasil. 2019. ( $\mathrm{N}=17$ ). ta entre 2013-2017. João Pessoa, Paraíba, Brasil. 2019. (N=192)

\begin{tabular}{ccccccc}
\hline Variáveis & \multicolumn{6}{c}{ Ano } \\
\cline { 1 - 4 } Anos estudados & $\mathbf{2 0 1 3}$ & $\mathbf{2 0 1 4}$ & $\mathbf{2 0 1 5}$ & $\mathbf{2 0 1 6}$ & $\mathbf{2 0 1 7}$ & Total \\
\hline 1 a 3 anos & $4(24 \%)$ & $1(6 \%)$ & $2(12 \%)$ & $1(6 \%)$ & - & 8 \\
4 a 7 anos & $1(6 \%)$ & - & $1(6 \%)$ & - & $2(12 \%)$ & 4 \\
8 a 11 anos & $1(6 \%)$ & $1(6 \%)$ & $1(6 \%)$ & - & $2(12 \%)$ & 5 \\
>12 anos & - & - & - & - & - & 0 \\
\hline & Fonte: MS/SVS/CGIAE - Sistema de Informações sobre Mortalidade - SIM
\end{tabular}


Observa-se que o somatório dos casos de mortalidade materna incidiu sobre as mulheres com menos anos de instrução educacional. Acredita-se que este dado evidencia a parcela populacional pobre como aquela que mais morre, considerando que o maior número de vítimas perpassa por mulheres que não possuem escolaridade satisfatória, condizentes com estrato social desfavorecido.

Na Tabela 3 é possível observar a prevalência de mortalidade materna de acordo com a cor/raça e estado civil entre os anos de 20132017. No que tange a tendência étnica, mulheres pardas morrem mais, seguidas das brancas. A miscigenação brasileira também reflete a et- nia da morte materna na Paraíba. Ressalta-se que a raça negra foi pouco indicada, levando a considerar que talvez a sociedade ainda carregue consigo o preconceito e estigma da negritude, o que pode refletir diretamente na cor autodeclarada pela população.

Em relação a situação conjugal, o somatório dos dados explicitou maior mortalidade entre mulheres solteiras. Infere-se que mulheres solteiras podem não ter uma rede de apoio tão fortalecida quanto mulheres com algum parceiro conjugal. Talvez o suporte familiar seja um elemento sine qua non para a redução do número de mortes entre mulheres gestantes e puérperas.

TABELA 3. Distribuição da mortalidade materna de mulheres paraibanas por cor/raça e estado civil entre 20132017. João Pessoa, Paraíba, Brasil. 2019. ( $N=298)$

\begin{tabular}{ccccccc}
\hline Variáveis & \multicolumn{7}{c}{ Ano } \\
\cline { 1 - 5 } Cor/raça & 2013 & 2014 & 2015 & 2016 & 2017 & Total \\
\hline Branca & $12(4,02 \%)$ & $5(1,67 \%)$ & $5(1,67 \%)$ & $3(1,00 \%)$ & $3(1 \%)$ & 28 \\
Preta & - & $1(0,33 \%)$ & - & $1(0,33 \%)$ & $3(1 \%)$ & 5 \\
Amarela & - & - & - & $1(0,33 \%)$ & - & 1 \\
Parda & $26(8,72 \%)$ & $25(8,38 \%)$ & $30(10,06 \%)$ & $43(14,42 \%)$ & $28(9,39 \%)$ & 152 \\
Indígena & - & - & $1(0,33 \%)$ & - & $2(0,67 \%)$ & 3 \\
\hline Estado civil & & & & & & \\
\hline Solteira & $14(4,69 \%)$ & $12(4,02 \%)$ & $7(2,34 \%)$ & $11(3,69 \%)$ & $14(4,69 \%)$ & 58 \\
Casada & $11(3,69 \%)$ & $9(3,02 \%)$ & $11(3,69 \%)$ & $12(4,02 \%)$ & $7(2,34 \%)$ & 50 \\
Viúva & - & - & $1(0,33 \%)$ & - & - & 1 \\
\hline
\end{tabular}

Segundo o último Censo Demográfico do ano de 2010, divulgado pelo Instituto Brasileiro de Geografia e Estatística (IBGE), os dados relacionados a cor/raça, quando comparados ao Censo anterior, mostram crescimento de $38,5 \%$ para $43,1 \%$ de população parda 9 .

O perfil epidemiológico dos óbitos maternos das gestantes atendidas em hospital público de referência em Recife-PE, com base nas declarações de óbitos, evidenciou que muIheres morrem mais na faixa etária entre $20 \mathrm{e}$ 29 anos, representando $47,3 \%$ dos casos. Em relação ao estado civil, foi verificado número elevado de registros ignorados (63,4\%). Dentre os dados válidos, as mães solteiras $(20,4 \%)$ prevaleceram sobre as casadas $(16,1 \%)$. Sobre a etnia, os óbitos prevaleceram entre as mulheres da raça negra $45,1 \%$, seguida da cor branca, perfazendo $20,4 \%$ do total de casos. ${ }^{10}$

As condições inadequadas de moradia, falta de saneamento básico, baixo nível de escolaridade e situações socioeconômicas precárias são fatores desencadeantes de morbidades e mortalidades na população. 
Logo, esse campo merece atenção imediata e constante dos órgãos públicos, considerando que o sistema de saúde brasileiro é focado na prevenção de doenças e redução de agravos em saúde. ${ }^{11}$

Nesse contexto, a atenção básica pode atuar na linha de frente para a prevenção da mortalidade materna por meio do Programa de Humanização do Pré-Natal e Nascimento (PHPN). O treinamento dos profissionais das unidades de saúde da família aliado ao conhecimento teórico sobre as principais causas diretas e indiretas de mortalidade materna poderia ser uma ferramenta salutar à prevenção das complicações decorrentes da gestação, parto e puerpério. $^{12}$

Com efeito, a pesquisa identificou que as mulheres que faziam pré-natal com o parceiro, tiveram maior sensação de segurança e amparo, demonstrando que a rede de apoio pode auxiliar na redução das intercorrências, complicações e desconfortos advindos da gestação ou pós-parto. ${ }^{13}$

As complicações, que surgem durante a gravidez, podem ter redução significativa através da realização de um bom pré-natal, o planejamento reprodutivo, o monitoramento.

\section{CONCLUSÕES}

Neste estudo, constatou-se que a morte materna por causas obstétricas diretas foi mais prevalente, em um contexto socioeconômico desfavorável a mulher, atingindo mulheres paraibanas com baixa escolaridade, pardas e sem parceria conjugal. O pré-natal qualificado e humanizado na atenção básica pode tornar as mulheres menos vulneráveis às complicações relacionadas à gestação, parto e puerpério. Para isso, é preciso que toda a equipe da atenção básica esteja interligada e
Com esses cuidados, o diagnóstico precoce e o tratamento eficaz das complicações tendem a reduzir os óbitos, possibilitando o direito fundamental do ser humano que é o direito à vida. ${ }^{14}$

Apesar das complicações apresentadas nas gestações permanecerem como grandes obstáculos para o bem-estar do binômio mãe-filho, um dos objetivos do milênio é a redução significativa da mortalidade materna, das desigualdades sociais e o aumento da qualidade da assistência obstétrica em todos os níveis de atenção, principalmente no nível primário. $\mathrm{A}$ meta para 2030 é reduzir a mortalidade materna para aproximadamente 20 mortes para cada 100 mil nascidos vivos. ${ }^{15}$

Essa taxa se apresenta de forma mais elevada nos lugares onde os fatores determinantes e condicionantes de saúde são poucos ou inexistentes. ${ }^{14}$ Por isso, mais importante que fiscalizar, é prover condições à redução de morbimortalidades, bem como munir a população de informação que seja capaz de mudar comportamentos, tornando a sociedade capaz de identificar possíveis problemas de saúde. Desse modo, a atuação precoce pode reduzir as causas evitáveis de morte.

unida para colocar em prática as políticas públicas de atenção à saúde da mulher e criança.

Os dados do sistema de informação não permitem associações estatísticas, tornando-se como limitação da pesquisa. Incentivam-se estudos mais robustos, bem como estratégias de educação em saúde e efetivação das políticas públicas que garantem a mulher o direito a uma atenção humanizada e qualificada durante o ciclo gravídico-puerperal. 


\title{
MATERNAL MORTALITY FOR OBSTETRIC CAUSES IN PARAÍBA STATE
}

\begin{abstract}
Maternal mortality is an aggravation that, despite being mostly due to preventable causes, still presents itself as a serious public health problem. This study aimed to analyze maternal mortality due to obstetric causes. This is an ecological, retrospective and quantitative study conducted in the period of June 2019. The study identified that maternal deaths are higher in direct causes, among the group of less literate, black and single women. There is a need for better professional assistance, in a qualified and holistic way, in view of the social vulnerability of women to the risk of maternal mortality. Thus, it was concluded that skilled prenatal care is a determining factor for the reduction of these maternal deaths due to obstetric causes in the state of Paraíba.
\end{abstract}

KEYWORDS: Women's health. Obstetrics. Public health. Nursing

\section{REFERÊNCIAS}

1. Marinho, ACN, Paes NA. Maternal mortality in the state of Paraíba Brazil: association between variables. Rev. Esc. Enferm. USP. 2015; 44(3):7227. Disponível em: <http://www.scielo.br/pdf/reeusp/v44n3/en_26.pdf >.

2. Dias JMG, Oliveira APS, Cipolotti R, Monteiro KKSM, Pereira RO. Mortalidade materna. Rev. Med. Minas Gerais. 2015; 25(2):173-9. Disponível em: <http://www.dx.doi.org/10.5935/22383182.2015003>.

3. Silva BGC, Lima NP, Silva SG, Antúnez SF, Seerig $L M$, Méndez $M C R$, et al. Mortalidade materna no Brasil no período de 2001 a 2012: tendência temporal e diferenças regionais. Rev. Bras. Epidemiol. 2016; 19(3):484-93. Disponível em: <https://www.scielosp.org/article/rbepid/2016. v19n3/484-493/pt>.

4. Baptista RS, Dutra MOM, Coura AS, Sousa FS. Assistência pré-natal: ações essenciais desenvolvidas pelos enfermeiros. Enferm. Glob. 2015; 40:112-27. Disponível em: <http://scielo.isciii.es/ pdf/eg/v14n40/pt_clinica5.pdf>.

5. Brasil. Ministério da Saúde. Resolução n. 466/2012: dispõe sobre pesquisas envolvendo seres humanos. Conselho Nacional de Saúde: Brasília, 2012. Disponível em <http:// bvsms.saude.gov.br/bvs/saudelegis/cns/2013/ res0466_12_12_2012.html>.

6. OPAS - Organização Pan-Americana de Saúde. Folha informativa - Mortalidade Materna. 2018. Disponível em: <https://www.paho.org/ bra/index.php?option=com_content\&view=article\&id=5741:folha-informativa-mortalidade-materna\&Itemid=820>.

7. Brasil. Ministério da Saúde. Protocolos da Atenção Básica: Saúde das Mulheres. Ministério da Saúde, Instituto Sírio-Libanês de Ensino e Pesquisa, Brasília: Ministério da Saúde, 2016. Disponível em: <http://bvsms.saude.gov.br/bvs/ publicacoes/protocolos_atencao_basica_saude_mulheres.pdf $>$. 
8. Mamede FV, Prudêncio PS. Contribuições de programas e políticas públicas para a melhora da saúde materna. Rev. Gaúcha Enferm. 2015; 36(esp):262-6. Disponível em: <www.scielo. br/pdf/rgenf/v36nspe/0102-6933-rgenf-36-spe-0262.pdf $>$.

9. IBGE - Instituto Brasileiro de Geografia e Estatística. Pesquisa Nacional por Amostra de Domicílios. Síntese dos Indicadores de 2009. Rio de Janeiro: IBGE; 2010. Disponível em: < https://biblioteca.ibge.gov.br/visualizacao/livros/liv45767. pdf $>$.

10. Menezes MLN, Bezerra JFO, Bezerra JFO. Perfil epidemiológico dos óbitos maternos em hospital de referência para gestação de alto-risco. Rev. Rene. 2015; 16(5): 714-21. Disponível em: <http:// www.redalyc.org/pdf/3240/324042637013.pdf>.

11. Leal SYP, Lima VLA, Silva AF, Soares PDFL, Santana LR, Pereira A. Percepção de enfermeiras obstétricas acerca da violência obstétrica. Cogitare Enferm. 2018; (23)2:e52473. Disponível em: <https://revistas.ufpr.br/cogitare/article/ view/52473/pdf $>$.
12. Rodrigues DP, Alves VH, Penna LHG, Pereira AV, Branco MBLR, Silva LA. A peregrinação no período reprodutivo: uma violência no campo obstétrico. Esc. Anna Nery. 2015; 19(4):614-20. Disponível em: <http://www.scielo.br/pdf/ean/ v19n4/1414-8145-ean-19-04-0614.pdf>.

13. Oliveira EC, Barbosa, SM, Melo SEP. A importância do acompanhamento pré-natal realizado por enfermeiros. Revista Científica Fac Mais. 2016; 7(3):25-38. Disponível em: <http:// revistacientifica.facmais.com.br/wp-content/ uploads/2017/01/Artigo-02-A-import\%C3\%A2ncia-do-acompanhamento-pr\%C3\%A9-natal-realizado-por-enfermeiros.pdf >.

14. Martins ACS, Silva LS. Epidemiological profile of maternal mortality. Rev. Bras. Enferm. 2018; 71(supl.1):677-83. Disponível em: <http://www. scielo.br/pdf/reben/v71s1/0034-7167-reben-71-s1-0677.pdf>.

15. ONU - Organização das Nações Unidas. Relatório Sobre os Objetivos de Desenvolvimento do Milênio. 2015. Disponível em: <https://www. unric.org/pt/images/stories/2015/PDF/MDG2015_ PT.pdf $>$. 